\title{
Design for Saving Energy Based On Energy Flow Analysis Method and CAE Technology
}

\author{
Wang Hong-lei ${ }^{1, a}$ Xiang Dong ${ }^{1, b}$ Duan Guang-hong ${ }^{1, c}$ \\ ${ }^{1}$ Department of Precision Instruments and Mechanology, Tsinghua University, Beijing, China

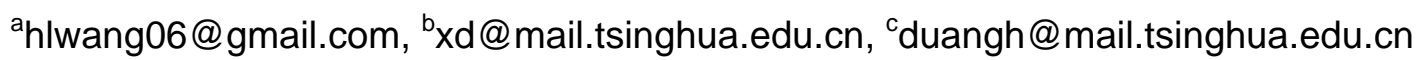

\section{Keywords: energy flow analysis; design for saving energy; CAE}

\begin{abstract}
This paper presents the design method of electromechanical products for saving energy based on energy flow analysis. The energy transfer model and energy correlation model are built through analyzing the relation of energy flow and products performance in order to address the conflict between energy saving and performance optimization. Based on the energy transfer model, the performance pertinences and performance margins of electromechanical products are analyzed with CAE technology and value engineering theory. Finally, the split air-conditioner's outdoor unit is studied and the design method for saving energy based on energy flow analysis is adopted to solve the conflict between air flow increasing and noise reduction.
\end{abstract}

\section{Introduction}

Energy and material saving has become one of the most important strategies for sustainable development in China. In recent years, the design method of electromechanical products has been attracting research focuses of some institutes and proper design method will play an important role in achieving energy saving. Generally speaking, functions of electromechanical products are usually achieved by energy acting on functional units, such as parts, components, assemblies, and so on. However, in many situations, the energy saving technology makes negative effects on the functions and performances of products. For example, increasing air flow of the split air-conditioner's outdoor unit is an important method to improve energy efficiency, but improper method of air flow increasing will cause unexpected performances, such as noise, vibration. Therefore, energy saving of electromechanical products must comprehensively consider the performances of products.

As a key factor to achieve the function and performance of electromechanical products, energy has been discussed in many design methods. Paul and Beitz[1]point out that products' function and performance can be achieved through the design of the product or system input and output (I/O), which is composed of the energy flow, material flow and the signal flow. Stone[2]introduces such a design language, called a functional basis, which is characterized in a verb-object format. The set of flow can describe the energy, material and signal which changes with the time. Gao and Huang[3, 4] presents that adequate and appropriate functional surface of energy is necessary for energy transfer or conversion during components and parts production. These functional surfaces can form a continuous and orderly energy chain to get the acting form of energy which is needed to achieve the desired function. However, these studies focus on how to achieve the function and performance of products; it's difficult to guide the energy saving design, because the design conflict often exists between the product performance and energy saving. Therefore, the design method for saving energy which is basis on energy flow analysis of electromechanical products is put forward in this paper.

\section{Energy flow analysis model for saving energy}

Firstly, a general F-E (Function-Energy) model for electromechanical products will be built to describe the relation between energy and products performances. Varied definitions of function have been represented in many academic papers. For example, Rodenacke[5]defines function as the 
relationship of input and output between energy, material and information. Stone[2]expresses function as an operation to be performed by a device or artifact. However, those definitions of function can't describe the degree of function achieving. So, in this paper, function is defined as the ability of completing with customer needs after some energy acting on functional units, and performance is defined to describe the degree of function completion. The F-E model can be used to form function space where we can look for function units which are related with energy. Based on the F-E model, performance constraint set is built to describe the situation of functional completion which is expressed as

$$
\Omega_{F C}=<P, S>
$$

The symbol $P$ represents a subset of the function space and $S$ is the set of constraint state for $P$. The energy flow model is different under the conditions of different performance constraint set. In order to make the $\Omega_{F C}$ have a quantitative expression, conversion of $\Omega_{F C}$ can be expressed as

$$
f: \Omega_{F C} \rightarrow \Gamma_{P I}
$$

$\Gamma_{P I}$ represents the set of the specific design parameters corresponds to one or some performance constraints. For example, if the energy efficiency improving and noise reducing for household air-conditioner is selected as the performance constraints, air flow or velocity fluctuation of outlet can be analyzed as the elements of $\Gamma_{P I}$ by the transformation relationship $f$. Based on $\Gamma_{P I}$, energy flow analysis of electromechanical products for energy saving can be accomplished by the following steps.

Energy transfer model (ETM). Usually different functions and performance have different realization forms of energy. For example, the energy of mechanical system can be represented by force, torque and velocity, but in the hydraulic or duck system we may use fluid pressure and volumetric flow to describe the realization form of energy. John Terninko[6]describes the energy-bearing relationship between two varied components in the Substance-Field (Su-field) Mode (shown in Fig. 1). In Fig. 1, the field which is an action or means of accomplishing the action is represented by $F_{\text {class }}$ and the substances which are objects of any level of complexity are represented by $\mathrm{S} 1$ and $\mathrm{S} 2$. Note that the desired function is the output from S1, caused by S2 with the help of $F_{\text {ckass }}$.

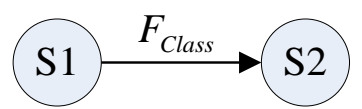

Fig. 1: Substance-Field (Su-field) Mode

However, for complex electromechanical product, it is not enough to describe the energy action of product's function and performance by the Su-field Mode. We must describe the relationship of energy transfer between different components, because varied functions and performances are often realized or guaranteed by different components of complex electromechanical products, and the relationship of energy transfer has diversity. So in this paper, energy flow element $\left(E_{s}\right)$ is defined to describe the energy transmission between functional components which is expressed as

$$
E_{s}=\left(I_{s}, O_{s}, T_{s}\right)
$$

The symbol $I_{s}$ represents the state of energy input, $O_{s}$ represents the state of energy output and $T_{s}$ describes the type of interface which includes Attachment(A), Transfer(T), Control \& Communication(C), Spatial(S), Field(F) and Environment(E) between different $E_{s}$. Each $E_{s}$ can be

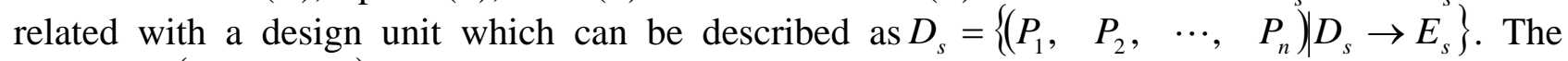
element $P_{i}(i=1,2 \cdots, n)$ is the specified structural parameter of the design unit. After the $E_{s}$ and $D_{s}$ are analyzed, the ETM can be built just like the Fig. 2 which is an ETM of air-conditioner. 


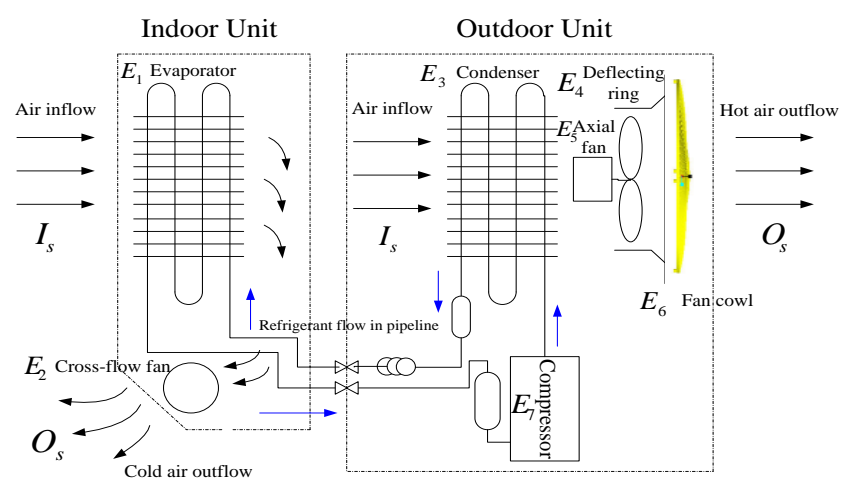

Fig. 2 Energy transfer model of air-conditioner

Energy correlation model (ECM). Energy Correlation Models (ECM) of components includes three sub-models, which are adjacency matrix of the components $\left(M_{A}\right)$, acting form of the energy $\left(M_{E A}\right)$ and energy state relationship $\left(M_{E S}\right)$. Each sub-model can be represented by a binary number, whose specific meaning is shown in the Table 1. For a given set of sub-models, the Energy Correlation Model may be expressed as $R_{E C M}=\left\{\begin{array}{lll}M_{A} & M_{E A} & M_{E S}\end{array}\right\}$. The symbol $R_{E C M}$ represents a matrix, in which each element is a three-dimensional vector (shown in Table 2).

Table 1: Specific Meaning of the Binary Number

\begin{tabular}{|c|c|c|c|}
\hline & 1 & 0 & -1 \\
\hline$M_{A}$ & Adjacency & Non-adjacency & \\
\hline$M_{E A}$ & Contact & Non-contact & \\
\hline$M_{E S}$ & Energy Output & None & Energy Input \\
\hline
\end{tabular}

Table 2: Expression Form of ECM

\begin{tabular}{|c|c|c|c|c|}
\hline Part No & P1 & P2 & $\cdots$ & PN \\
\hline P1 & & $\{1,1,1\}$ & $\cdots$ & $\{\mathbf{0 , 0 , 0}\}$ \\
\hline P2 & $\{1,-1,1\}$ & & $\cdots$ & $\{\mathbf{0 , 0 , 0}\}$ \\
\hline$\cdots$ & $\cdots$ & $\cdots$ & & $\cdots$ \\
\hline PN & $\{\mathbf{0 , 0 , 0}\}$ & $\{\mathbf{0 , 0 , 0}\}$ & $\cdots$ & \\
\hline
\end{tabular}

Analysis model for performance pertinence (PPM).In order to authentically guide the energy saving design of electromechanical products, it is not enough to rely on the ETM and ECM. So the PPM can be built which can quantitatively analyze the influence degree of parts for varied performances and is similar to the optimization model. The optimization goal can be described as

$\min -E=\sum E_{i}(i=1,2, \cdots, n)$

The symbol $\sum$ represents the sum of interaction between different $E_{s}$ that is the whole process of energy flow. Then we can find the important $E_{s}$ in the constraint of $\Gamma_{P I}$ and calculate the weight of $E_{s}$ which is defined as performance pertinence (PP). PP of components can be obtained by expert evaluation and the CAE analysis.

Analysis model for performance margins (PMM). Components of complex electromechanical products often take on varied functions and performances, but it is difficult to synthetically consider all of the performances during the detailed design stage of components. Based on the value engineering theory[7], the cost of some components for improving performance may be very high although components optimization can improve the performances such as energy saving. Therefore, in the process of design for energy saving of electromechanical products based on $\Gamma_{P I}$, we define performance margin (PM) as $P M=P / C$ where symbol $P$ represents the range of performance improving and $C$ represents the cost of performance improving. Then, PMM can be described as

$\max \quad P M=\sum P_{i} / C_{i}(i=1,2, \cdots, n)$

which experimental test and CAE analysis for various performances can be utilized to analyze PM of the components which have larger PP. 


\section{Application examples}

As an example application of the energy flow analysis method, the split air-conditioner's outdoor unit is analyzed. The energy flow for the split air-conditioner's outdoor unit is expressed by flow velocity, when we care the energy efficiency and noise reduction of air-conditioner, the element of $\Gamma_{P I}$ can be set air flow and velocity fluctuation. According to the PPM, air flow increasing is the optimization goal and the noise reduction is the constraint condition, the CAE model of the split air-conditioner's outdoor unit is built to calculate the air flow and velocity fluctuation (shown in Fig.3).

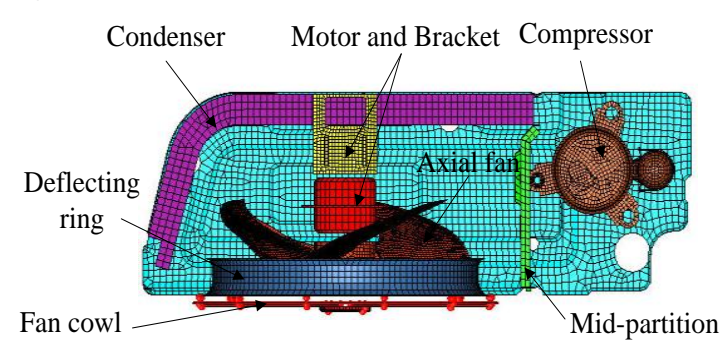

Fan cow
Fig.

3 CAE model of split air-conditioner's outdoor unit

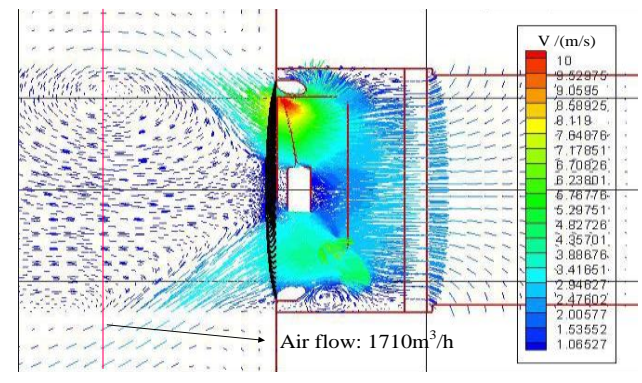

Fig. 4 Calculation result of the CAE model

Based on the calculation result (shown in Fig.4) and the expert evaluation method, we can find the components whose PP is larger such as axial fan, deflecting ring and the fan cowl. Based on the PMM, the PM of fan cowl is larger than the other two. So we determine the fan cowl as the optimization object for energy saving. After that fan cowl is improved by construct optimization, as the result the air flow increases $4.7 \%$ and noise value reduces $2.4 \%$. Thus, air flow increase and noise reduction are balanced effectively.

\section{Conclusion}

This paper describes a green design method for energy saving of electromechanical products based on energy flow model and CAE technology. The method provides a common procedure that can be used to solve the design conflict between the product performance and energy saving. By analyzing the energy flow models, performance pertinences and margins, the performances optimization and energy saving could be balanced. Based on the example of air-conditioner's outdoor design, the design method based on energy flow and CAE technology is effective to achieve energy saving of electromechanical products.

\section{References}

[1] G. Pahl and W. Beitz: Engineering design: a systematic approach (Springer, London 1996).

[2] R. B. Stone and K. L. Wood: "Development of a functional basis for design," Journal of Mechanical Design, Vol.122 (2000), pp. 359-370.

[3] C. Q. Gao, K. Z. Huang and Y. Zhang: "Research and Implementation on Conceptual Design Automation for Mechanical Products Based on Energy Transforming," China Mechanical Engineering, Vol.18 (2007), pp. 732-738.

[4] C. J. Li and K. Z. Huang: "Research on energy chain of conceptual design," Journal of PLA University of Science and Technology, Vol.9 (2008), pp. 264-268.

[5] W. G. Rodenacker: Methodisches Konstruieren (Springer-Verlag, Berlin 1976).

[6] T. John: "Su-Field Analysis," TRIZ Journal, Vol.2 (2000), pp. 1-12.

[7] L. D. Miles: Techniques of value analysis and engineering (McGraw-Hill, New York 1972). 